\title{
Integral Means of Univalent Solution for Fractional Differential Equation
}

\author{
Rabha W. Ibrahim, Maslina Darus \\ School of Mathematical Sciences, Faculty of Science and Technology, University Kebangsaan Malaysia, \\ Bangi, Malaysia \\ Email: rabhaibrahim@yahoo.com,maslina@ukm.my
}

Received June 12, 2011; revised April 20, 2012; accepted April 27, 2012

\begin{abstract}
By employing the Srivastava-Owa fractional operators, we consider a class of fractional differential equation in the unit disk. The existence of the univalent solution is founded by using the Schauder fixed point theorem while the uniqueness is obtained by using the Banach fixed point theorem. Moreover, the integral mean of these solutions is studied by applying the concept of the subordination.
\end{abstract}

Keywords: Fractional Calculus; Subordination; Superordination; Univalent Solution; Fractional Differential Equation; Integral Mean

\section{Introduction}

Recently, the theory of fractional calculus has found interesting applications in the theory of analytic functions. The classical definitions of fractional operators and their generalizations have fruitfully been applied in obtaining, for example, the characterization properties, coefficient estimates [1], distortion inequalities [2] and convolution structures for various subclasses of analytic functions and the works in the research monographs. In [3], Srivastava and Owa, gave definitions for fractional operators (derivative and integral) in the complex z-plane $\mathbb{C}$ as follows:

Definition 1.1. The fractional derivative of order $\alpha$ is defined, for a function $f(z)$ by

$$
D_{z}^{\alpha} f(z):=\frac{1}{\Gamma(1-\alpha)} \frac{\mathrm{d}}{\mathrm{d} z} \int_{0}^{z} \frac{f(\zeta)}{(z-\zeta)^{\alpha}} \mathrm{d} \zeta ; 0 \leq \alpha<1,
$$

where the function $f(z)$ is analytic in simply-connected region of the complex z-plane $\mathbb{C}$ containing the origin and the multiplicity of $(z-\zeta)^{-\alpha}$ is removed by requiring $\log (z-\zeta)$ to be real when $(z-\zeta)>0$.

Definition 1.2. The fractional integral of order $\alpha$ is defined, for a function $f(z)$, by

$$
I_{z}^{\alpha} f(z):=\frac{1}{\Gamma(\alpha)} \int_{0}^{z} f(\zeta)(z-\zeta)^{\alpha-1} \mathrm{~d} \zeta ; \alpha>0,
$$

where the function $f(z)$ is analytic in simply-connected region of the complex z-plane $(\mathbb{C})$ containing the origin and the multiplicity of $(z-\zeta)^{\alpha-1}$ is removed by requiring $\log (z-\zeta)$ to be real when $(z-\zeta)>0$.

\section{Remark 1.1.}

$$
D_{z}^{\alpha}\left\{z^{\mu}\right\}=\frac{\Gamma(\mu+1)}{\Gamma(\mu-\alpha+1)}\left\{z^{\mu-\alpha}\right\}, \mu>-1 ; 0 \leq \alpha<1
$$

and

$$
I_{z}^{\alpha}\left\{z^{\mu}\right\}=\frac{\Gamma(\mu+1)}{\Gamma(\mu+\alpha+1)}\left\{z^{\mu+\alpha}\right\}, \mu>-1 ; \alpha>0 .
$$

Further properties of these operators can be found in $[4,5]$.

\section{Preliminaries}

Let $\mathcal{A}$ be the class of all normalized analytic functions

$$
f(z)=z+\sum_{n=2}^{\infty} a_{n} z^{n}
$$

in the open unit disk $U:=\{z \in \mathbb{C}:|z|<1\}$ satisfying $f(0)=0$ and $f^{\prime}(0)=1$. Let $\mathcal{H}$ be the class of analytic functions in $U$ and for any $a \in \mathbb{C}$ and $n \in \mathbb{N}$, $\mathcal{H}[a, n]$ be the subclass of $\mathcal{H}$ consisting of functions of the form $f(z)=a+a_{n} z^{n}+\cdots$.

For given two functions $F$ and $G$, which are analytic in $U$, the function $F$ is said to be subordinate to $G$ in $U$ if there exists a function $h$ analytic in $U$ with

$$
h(0)=0 \text { and }|h(z)|<1 \text { for all } z \in U
$$

such that

$$
F(z)=G(h(z)) \text { for all } z \in U .
$$

We denote this subordination by $F \prec G$. If $G$ is uni- 
valent in $U$, then the subordination $F \prec G$ is equivalent to $F(0)=G(0)$ and $F(U) \subset G(U)$.

Lemma 2.1 [6]. If the functions $f$ and $g$ are analytic in $U$ then

$$
\begin{aligned}
g(z) & \prec f(z) \Rightarrow \int_{0}^{2 \pi}\left|g\left(r e^{i \theta}\right)\right|^{\mu} \mathrm{d} \theta \\
& \leq \int_{0}^{2 \pi}\left|f\left(r e^{i \theta}\right)\right|^{\mu} \mathrm{d} \theta, \mu>0,0<r<1 .
\end{aligned}
$$

Lemma 2.2 [7]. Let $f, g$ be analytic function in $U$. Assume that $I_{z}^{\beta} g(z) \in \mathcal{H}[0,1], \quad I_{z}^{\alpha} f(z) \in Q(a)$ and

$$
\begin{aligned}
I_{z}^{\alpha} f(z)\left[1+\frac{z f^{\prime}(z)}{f(z)}\right] \text { is univalent in } U \text {. If } \\
\frac{|g(z)|+\left|g^{\prime}(z)\right|}{\Gamma(\beta)}<\frac{|f(z)|+\left|f^{\prime}(z)\right|}{\Gamma(\alpha)},
\end{aligned}
$$

then $q(z):=I_{z}^{\beta} g(z) \prec p(z):=I_{z}^{\alpha} f(z)$.

Our work is organized as follows: In Section 2, we will derive the integral means for normalized analytic functions involving fractional integral in the open unit $\operatorname{disk} U$

$$
p(z):=I_{z}^{\alpha} f(z) \prec q(z):=I_{z}^{\beta} g(z), \alpha, \beta \in(0,1) .
$$

In Section 3, we study the existence of locally univalent solution for the fractional diffeo-integral equation

$$
D_{z}^{\alpha} u(z)=h\left(z, u(z), k\left(z, I_{z}^{\beta} u(z)\right)\right), \alpha, \beta \in(0,1),
$$

subject to the initial condition $u(0)=0$, where $u: U \rightarrow \mathbb{C}$ is an analytic function for all $z \in U$ and $k: U \times \mathbb{C} \rightarrow \mathbb{C}, \quad h: U \times \mathbb{C} \times \mathbb{C} \rightarrow \mathbb{C}$ are analytic univalent functions in $z \in U$. The existence is shown by using Schauder fixed point theorem while the uniqueness is verified by using Banach fixed point theorem.

For that purpose we need the following definitions and results:

Let $M$ be a subset of Banach space $X$ and $A: M \rightarrow M$ an operator. The operator $A$ is called compact on the set $M$ if it carries every bounded subset of $M$ into a compact set. If $A$ is continuous on $M$ (that is, it maps bounded sets into bounded sets) then it is said to be completely continuous on $M$. A mapping $A: X \rightarrow X$ is said to be a contraction if there exists a real number $\rho, 0 \leq \rho<1$ such that

$$
\|A x-A y\| \leq \rho\|x-y\|, \text { for all } x, y \in X .
$$

Theorem 2.1. Arzela-Ascoli let $E$ be a compact metric space and $\mathcal{C}(E)$ be the Banach space of real or complex valued continuous functions normed by

$$
\|f\|:=\sup _{t \in E}|f(t)| \text {. }
$$

If $A=\left\{f_{n}\right\}$ is a sequence in $\mathcal{C}(E)$ such that $\underline{f}_{n}$ is uniformly bounded and equi-continuous, then $\bar{A}$ is compact.

Theorem 2.2. (Schauder) Let $X$ be a Banach space, $M \subset X$ a nonempty closed bounded convex subset and $P: M \rightarrow M$ is compact. Then $P$ has a fixed point.

Theorem 2.3. (Banach) If $X$ is a Banach space and $P: X \rightarrow X$ is a contraction mapping then $P$ has a unique fixed point.

\section{Existence and Uniqueness}

In this section, we established the existence and uniqueness solution for the diffeo-integral Equation (1). Let $\mathcal{B}:=\mathcal{C}[U, \mathbb{C}]$ be a Banach space of all continuous functions on $U$ endowed with the sup. norm

$$
\|u\|:=\sup _{z \in U}|u(z)| \text {. }
$$

Lemma 3.1. If the function $h$ is analytic, then the initial value problem (1) is equivalent to the nonlinear Volterra integral equation

$$
u(z)=\int_{0}^{z} \frac{(z-\zeta)^{\alpha-1}}{\Gamma(\alpha)} h(\zeta, u(\zeta), v(\zeta)) \mathrm{d} \zeta ; \alpha \in(0,1) .
$$

In other words, every solution of the Volterra Equation (2) is also a solution of the initial value problem (1) and vice versa.

The following assumptions are needed in the next theorem:

(H1) There exists a continuous function $\rho(z)$ on $U$ and increasing positive function $\Psi \in C\left[\mathbb{R}_{+}, \mathbb{R}_{+}\right]$such that

$$
|h(z, u, v)| \leq|\rho(z)| \Psi(\|u\|+\|v\|)
$$

with the property that

$$
\Psi(a(z)\|u\|+b(z)\|v\|) \leq a(z) \Psi(\|u\|)+b(z) \Psi(\|v\|) .
$$

Note that $C\left[\mathbb{R}_{+}, \mathbb{R}_{+}\right]$is the Banach space of all continuous positive functions.

(H2) There exists a continuous function $p$ in $U$, such that

$$
\left|k\left(z, I^{\beta} u(z)\right)\right| \leq \frac{\|p\|}{\Gamma(\beta+1)}\|u\| .
$$

Remark 3.1. By using fractional calculus we observe that Equation (2) is equivalent to the integral equation of the form

$$
u(z)=I_{z}^{\alpha} h\left(z, u(z), k\left(z, I_{z}^{\beta} u(z)\right)\right)
$$

that is, the existence of Equation (2) is the existence of the Equation (3).

Theorem 3.1. Let the assumptions (H1) and (H2) hold. Then Equation (1) has a univalent solution $u(z)$ on $U$.

Proof. We need only to show that $P: \mathcal{B} \rightarrow \mathcal{B}$ has a fixed point by using Theorem 1.2 where 


$$
\begin{aligned}
& (P u)(z):=I_{z}^{\alpha} h\left(z, u(z), I_{z}^{\beta} k(z, u(z))\right) \text {, then } \\
& |(P u)(z)|=\left|I_{z}^{\alpha} h\left(z, u(z), k\left(z, I_{z}^{\beta} u(z)\right)\right)\right| \\
& \leq I_{z}^{\alpha}\left|h\left(z, u(z), k\left(z, I_{z}^{\beta} u(z)\right)\right)\right| \\
& \leq \frac{\|\rho\|}{\Gamma(\alpha+1)} \Psi\left(\|u\|+\left|k\left(z, I_{z}^{\beta} u(z)\right)\right|\right) \\
& \leq \frac{\bar{\Psi}\|\rho\|}{\Gamma(\alpha+1)}\left[1+\frac{\|\rho\|}{\Gamma(\beta+1)}\right] \\
& =\frac{\bar{\Psi}\|\rho\|[\Gamma(\beta+1)+\|\rho\|]}{\Gamma(\beta+1) \Gamma(\alpha+1)}
\end{aligned}
$$

where $\bar{\Psi}:=\sup _{u \in \mathcal{B}} \Psi(\|u\|)$. Thus we obtain that

$$
\|P\| \leq \frac{\bar{\Psi}\|p\|[\Gamma(\beta+1)+\|p\|]}{\Gamma(\beta+1) \Gamma(\alpha+1)}=: r
$$

that is $P: B_{r} \rightarrow B_{r}$. Then $P$ mapped $B_{r}$ into itself. Now we proceed to prove that $P$ is equicontinuous. For $z_{1}, z_{2} \in U$ such that $z_{1} \neq z_{2},\left|z_{2}-z_{1}\right|<\delta, \quad \delta>0$. Then for all $u \in S$, where

$$
S:=\left\{u \in \mathbb{C},:|u| \leq \frac{\bar{\Psi}\|\rho\|[\Gamma(\beta+1)+\|p\|]}{\Gamma(\beta+1) \Gamma(\alpha+1)}\right\},
$$

we obtained

$$
\begin{aligned}
& \left|(P u)\left(z_{1}\right)-(P u)\left(z_{2}\right)\right| \\
& =\mid \int_{0}^{z_{1}} \frac{\left(z_{1}-\zeta\right)^{\alpha-1}}{\Gamma(\alpha)} h(\zeta, u(\zeta), v(\zeta)) \mathrm{d} \zeta \\
& \quad-\int_{0}^{z_{2}} \frac{\left(z_{2}-\zeta\right)^{\alpha-1}}{\Gamma(\alpha)} h(\zeta, u(\zeta), v(\zeta)) \mathrm{d} \zeta \mid \\
& \leq \frac{\|h\|}{\Gamma(\alpha)} \mid \int_{0}^{z_{1}}\left[\left(z_{1}-\zeta\right)^{\alpha-1}-\left(z_{2}-\zeta\right)^{\alpha-1}\right] \mathrm{d} \zeta \\
& \quad+\int_{z_{1}}^{z_{2}}\left(z_{2}-\zeta\right)^{\alpha-1} \mathrm{~d} \zeta \mid \\
& =\frac{\|h\|}{\Gamma(\alpha+1)}\left|\left[2\left(z_{2}-z_{1}\right)^{\alpha}+z_{2}^{\alpha}-z_{1}^{\alpha}\right]\right| \\
& \leq 2 \frac{\|h\|}{\Gamma(\alpha+1)}\left|z_{2}-z_{1}\right|^{\alpha} \leq 2 \frac{\|h\|}{\Gamma(\alpha+1)} \delta^{\alpha},
\end{aligned}
$$

which is independent of $u$.

Hence $P$ is an equicontinuous mapping on $S$. Moreover, for $z_{1} \neq 0, \quad z_{2} \neq 0 \in U$ such that $z_{1} \neq z_{2}$ and under assumption (H1), we show that $P$ is a univalent function. The Arzela-Ascoli theorem yields that every sequence of functions $\left\{u_{n}\right\}$ from $P(S)$ has a uniformly convergent subsequence, and therefore $P(S)$ is relatively compact. Schauder's fixed point theorem asserts that $P$ has a fixed point. The univalency of the function $h$ yields that $u$ is a univalent solution.

Now we discuss the uniqueness solution for the problem (1). For this purpose let us state the following assumptions:

(H3) Assume that there exists a positive number $L$ such that for each $u_{1}, v_{1}$ and $u_{2}, v_{2} \in \mathcal{B}$,

$$
\begin{aligned}
& \left|h\left(z, u_{1}(z), v_{1}(z)\right)-h\left(z, u_{2}(z), v_{2}(z)\right)\right| \\
& \leq L\left[\left\|u_{1}-u_{2}\right\|+\left\|v_{1}-v_{2}\right\|\right] .
\end{aligned}
$$

(H4) Assume that there exists a positive number $\ell$ such that for each $u_{1}, u_{2} \in \mathcal{B}$ we have

$$
\left|k\left(z, I_{z}^{\beta} u_{1}(z)\right)-k\left(z, I_{z}^{\beta} u_{2}(z)\right)\right| \leq \frac{\ell}{\Gamma(\beta+1)}\left\|u_{1}-u_{2}\right\| .
$$

Theorem 3.2. Let the hypotheses $(\mathrm{H} 1-\mathrm{H} 4)$ be satisfied. If $\frac{L[\Gamma(\beta+1)+\ell]}{\Gamma(\beta+1) \Gamma(\alpha+1)}<1$, then (1) admits a unique univalent solution $u(z)$.

Proof. Assume the operator $P$ defined in Equation (4), we only need to show that $P$ is a contraction mapping that is $P$ has a unique fixed point which is corresponding to the unique solution of the Equation (1). Let $u_{1}, u_{2} \in \mathcal{B}$, then for all $z \in U$, we obtain that

$$
\begin{aligned}
& \left|\left(P u_{1}\right)(z)-\left(P u_{2}\right)(z)\right| \\
\leq & I_{z}^{\alpha} \mid h\left(z, u_{1}(z), k\left(z, I_{z}^{\beta} u_{1}(z)\right)\right) \\
& -h\left(z, u_{2}(z), k\left(z, I_{z}^{\beta} u_{2}(z)\right)\right) \mid \\
\leq & \frac{L}{\Gamma(\alpha+1)}\left[\left\|u_{1}-u_{2}\right\|+\left\|k\left(z, I_{z}^{\beta} u_{1}(z)\right)-k\left(z, I_{z}^{\beta} u_{2}(z)\right)\right\|\right] \\
\leq & \frac{L}{\Gamma(\alpha+1)}\left[\left\|u_{1}-u_{2}\right\|+\frac{\ell}{\Gamma(\beta+1)}\left\|u_{1}-u_{2}\right\|\right] \\
= & \frac{L[\Gamma(\beta+1)+\ell]}{\Gamma(\beta+1) \Gamma(\alpha+1)}\left\|u_{1}-u_{2}\right\| .
\end{aligned}
$$

Thus by the assumption of the theorem we have that $P$ is a contraction mapping. Then in view of Banach fixed point theorem, $P$ has a unique fixed point which corresponds to the univalent solution (Theorem 3.1) of Equation (1). Hence the proof.

The next result shows the integral means of univalent solutions of problem (1).

Theorem 3.3. Let $u_{1}(z), u_{2}(z)$ be two analytic univalent solutions for the Equation (1) satisfying the assumptions of Lemma 2.2 with $p(z):=u_{1}(z)$ and $q(z):=u_{2}(z)$ then

$$
\int_{0}^{2 \pi}\left|q\left(r e^{i \theta}\right)\right|^{\mu} \mathrm{d} \theta \leq \int_{0}^{2 \pi}\left|p\left(r e^{i \theta}\right)\right|^{\mu} \mathrm{d} \theta, \mu>0,0<r<1 .
$$


Proof. Setting $f(z):=h\left(z, u_{1}, v_{1}\right), g(z):=h\left(z, u_{2}, v_{2}\right)$, Lemma 2.2 implies that $q(z) \prec p(z)$. Hence in view of Lemma 1.2, we obtain the result.

Example 3.1. Consider the fractional problem

$$
D_{z}^{1 / 2} u(z)=\frac{u(z)+I_{z}^{1 / 2} u(z)}{10},
$$

where $u: U \rightarrow U$ and $k\left(z, I_{z}^{\beta} u(z)\right)=I_{z}^{1 / 2} u(z)$. We observe that $\Psi=u(z)+I_{z}^{1 / 2} u(z)$ and $\bar{\Psi}=2.13$ and

$$
r=\frac{\bar{\Psi}\|p\|[\Gamma(3 / 2)+\|p\|]}{\Gamma(3 / 2) \Gamma(3 / 2)}=0.52,
$$

where $\rho(z)=\frac{1}{10}$ and $p(z)=1$. Thus in view of Theorem 3.1, the problem (5) has a solution in the unit disk.

\section{REFERENCES}

[1] M. Darus and R. W. Ibrahim, "Radius Estimates of a Subclass of Univalent Functions," Matematicki Vesnik, Vol. 63, No. 1, 2011, pp. 55-58.

[2] H. M. Srivastava, Y. Ling and G. Bao, "Some Distortion
Inequalities Assotiated with the Fractional Drivatives of Analytic and Univalent Functions," Journal of Inequalities in Pure and Applied Mathematics, Vol. 2, No. 2, 2001, pp. 1-6.

[3] H. M. Srivastava and S. Owa, "Univalent Functions, Fractional Calculus, and Their Applications," Halsted Press, John Wiley and Sons, New York, 1989.

[4] R. W. Ibrahim and M. Darus, "Subordination and Superordination for Analytic Functions Involving Fractional Integral Operator," Complex Variables and Elliptic Equations, Vol. 53, No. 11, 2008, pp. 1021-1031. doi:10.1080/17476930802429131

[5] R. W. Ibrahim and M. Darus, "Subordination and Superordination for Univalent Solutions for Fractional Differential Equations," Journal of Mathematical Analysis and Applications, Vol. 345, No. 2, 2008, pp. 871-879. doi:10.1016/j.jmaa.2008.05.017

[6] J. E. Littlwood, "On Inequalities in the Theory of Functions," Proceedings of the London Mathematical Society, Vol. 23, 1925, pp. 481-519. doi: $10.1112 / \mathrm{plms} / \mathrm{s} 2-23.1 .481$

[7] R. W. Ibrahim and M. Darus, "Integral Means of Univalent Solution for Fractional Equation in Complex Domain," Acta Universitatis Apulensis, Vol. 23, 2010, pp. $1-8$. 